\title{
Modeling of stressed deformed state of lattice anisogrid constructions
}

\author{
Valeriy O. Kaledin \\ Novokuznetsk branch institute \\ Kemerovo state university \\ Novokuznetsk, Russian Federation \\ vkaled@mail.ru
}

\author{
Tatyana V. Burnysheva \\ Novokuznetsk branch institute \\ Kemerovo state university \\ Novokuznetsk, Russian Federation \\ tburn@mail.ru
}

\author{
Olga A. Shteinbreher \\ Novokuznetsk branch institute \\ Kemerovo state university \\ Novokuznetsk, Russian Federation \\ olga_sht@mail.ru
}

\begin{abstract}
The use of discrete modeling and the mathematical model of joint deformation to calculate the stress-strain state of the structure is considered in the work. The authors cite the model of joint deformation and the procedure for constructing a discrete model. As an example, the calculation of the stress-strain state of a set anisogrid structure with notches and gains is considered, and an analysis of stress distribution in structural elements is given. The formal formulation of optimal and rational design problems is described using this approach.
\end{abstract}

Keywords - mathematical modeling; discrete model; lattice anisogrid shells; joint deformation; optimal design; rational design

\section{INTRODUCTION}

In the designs of current spacecraft (SC) and launch vehicle, anisogrid lattice structures made of composite materials produced by the continuous winding method are increasingly used [1]. The main classification feature of the lattice construction is the presence of several kinds of intersecting rib that form a regular structure. Anisogrid structures are used as independent structural elements connecting various aggregates (for example, output unit with carrier) or together with the skin, providing a solid surface for sealing or protecting the units from the effects of vacuum or air flow (Fig. 1).

The existence of cutouts and reinforcing elements leads to a variety of structural-power circuits of anisogrid structures in various products. This makes it difficult and complicates the solution of practical problems to ensure the strength of the compartments of spacecraft and launch vehicles [2].
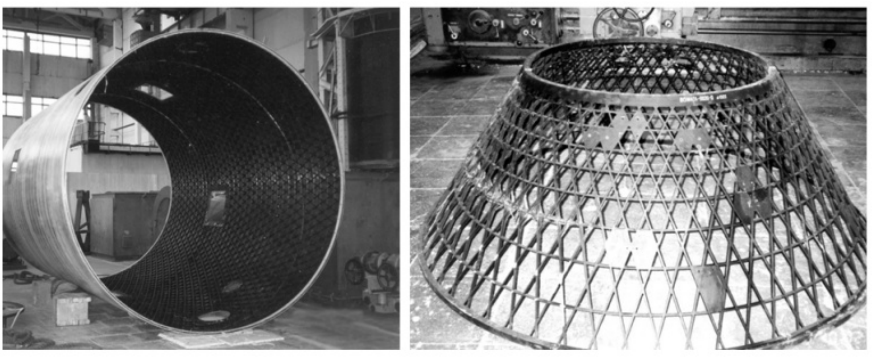

Fig. 1. Anisogrid shell constructions made of composite materials

\section{THE TOPICALITY OF MODELING ANISOGRID STRUCTURES}

The production technology of anisogrid lattice structures made of polymer composite materials appeared in the late 1970s and was developed mainly thanks to the works of the school of the Academician of RAS V.V. Vasilyev. By now, a lot of experience the early $2000 \mathrm{~s}$, advanced methods for the optimal design of large-sized highly loaded anisogrid structures of irregular structure (having a large number of structural cutouts and nodes that transmit local loads) [2]. This allowed to further improve their mass characteristics.

But the variety of topology requires an individual approach to mesh structures depending on their structure. Therefore, it is not possible to use standard solutions in all cases. A laborious calculation of the stress-strain state of a structure using numerical methods and program complexes is required to ensure the strength of the product. Optimization methods for strength, stiffness and stability based on the continuum model are developed for anisogrid structures of a regular structure. Whereas a violation of regularity (such as cutouts) can only be taken into account when discrete modeling of the construction. Multiple calculation of families of the same type of 
construction is necessary to ensure strength, for example, by the finite element method $[10,11]$. Since such a calculation has a high labor intensity and duration, the development of improved methods and mathematical models is necessary. These methods and models should preserve the advantages of discrete modeling and allow us to obtain sufficiently complete data on stresses and deformations when varying the design parameters.

\section{MATHEMATICAL MODEL OF JOINT DEFORMATION}

The developed mathematical model of deformation of the anisogrid lattice structure is based on the idea of joint deformation of the rib structure and skin, if any. For this, the following kinematic hypotheses were used.

1. The deformation of the skin corresponds to the classical Kirchhoff-Love hypothesis: the material normal coincides with the geometric one, does not warp and does not change its length, that is, the deformations of the normal and transverse shifts are absent [12].

2. Each edge is represented as a set of short beams. The beams have a section height commensurate with the length of the beam. The deformation of the beam corresponds to the hypothesis of Timoshenko: the cross-sections of the beam do not change the shape and dimensions, but do not remain perpendicular to the curved axis of the beam [13].

3. The displacements of the points of the beams of different kinds (spiral, annular) at their intersections are continuous and identical.

4. The movements of the ribs and the skin coincide on the lines of their contact surface, which run parallel to the axes of the ribs.

We consider the mutual arrangement of the geometric normal to the reduction surface and the material normals for the skin and the rib structure to represent the joint deformation of the shell and the rib structure.

The scheme of joint deformation of the shell and rib structure is shown in Fig. 2. The material normal in the shell is the same as the geometric one: it rotates from the initial position to an angle $\theta^{\prime}$ at deformation. In the edge, the material normal rotates in space by an angle $\theta$. The angle $\gamma$ between the geometric and material normal is equal to the strain of the transverse shear.

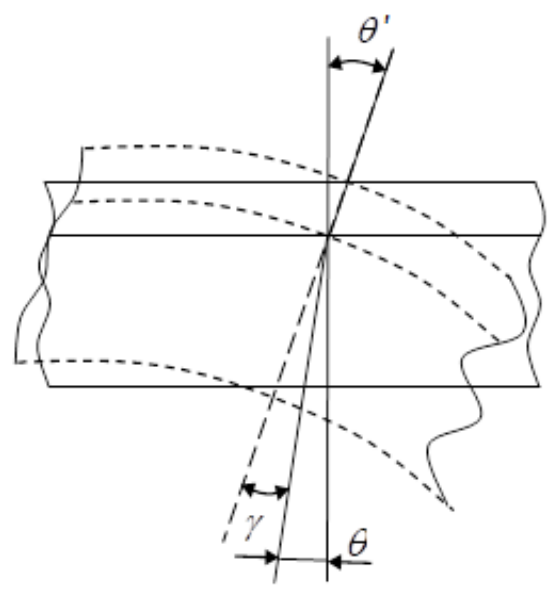

Fig. 2. Joint deformation of the skin and rib structure: - - - geometric normal, _ material normal; $\theta^{\prime}$ - angle of rotation of the geometric normal to the skin, $\theta$ - angle of rotation of the material normal to the edges, $\gamma$ - shear angle

The kinematic hypothesis for beams ignores the deformation section. Therefore, the rotation of the beam relative to its longitudinal axis must be zero when there is equality of movement of the rib and beam on the contact surface. This is unacceptable since the height of the cross section exceeds the width by a factor of 10 or more. Thus, the weaker condition of equality of displacements on the contact line can be considered as the assumption of "in stock" in hardness. It is more rational for the description of kinematics than the assumption of the coincidence of displacements on the entire contact surface (it leads to an overestimation of hardness).

The field of displacements of the lattice shell is assumed to be continuous. Let the surface of the contact be the surface of the contact of the ribs with the skin (Fig. 3). The axes of the main coordinate system $\left(s^{\prime}, t^{\prime}, n^{\prime}\right)$ are given at the nodes of the reduction surface. The axis $s^{\prime}$ is tangential to the shell. The axis $t$ ' is directed along the generatrix from the trailing edge to the front. The axis $n^{\prime}$ is directed along the outward normal. The movements and external forces will relate to the main system.

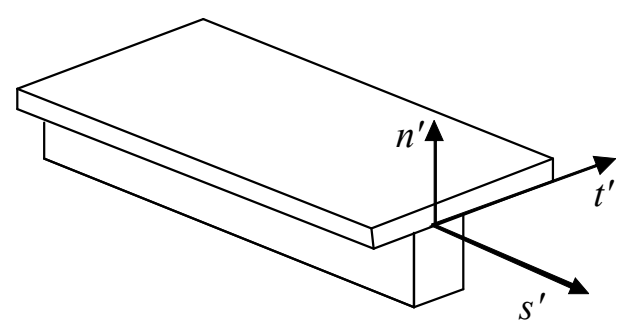

Fig. 3. Reduction surface

We introduce an auxiliary cylindrical coordinate system $(z$, $\varphi, r)$ to ensure the compatibility of movements. The $z$ axis coincides with the axis of the shell, angle $\varphi$ is measured from an arbitrarily chosen generator and the $r$-axis is directed along the outer normal to the reduction surface. Each point of the construction can be defined by the coordinates $(z, \varphi, n)$, where 
the coordinate $\mathrm{n}$ on the reduction surface is zero. The reduction surface in a cylindrical system is given by $r=r(z)$.

The normal coordinate of the point can be expressed in terms of the axial and radial coordinates:

$$
n(z, r)=\left(r-r_{0}(z)\right) / \sqrt{1+\left(\frac{d r_{0}}{d z}\right)^{2}}
$$

where $r_{0}=r_{0}(z)$ is the equation of meridian. This allows us to go from the parameterization $(z, r, \varphi)$ to the variables $(z, n, \varphi)$. The vector field of displacements in the form of a bundle of functions will have the form:

$$
\rho_{(z, n, \varphi)} \rho_{0}(z, \varphi)+n \cdot\left\{\begin{array}{l}
\rho_{+}(z, \varphi), n>0 \\
\rho_{-}(z, \varphi), n<0
\end{array},\right.
$$

where $u_{0}(z, \varphi)$ - displacement vector of the point of the reduction surface, $\psi^{+}$and $\psi^{-}-$the normal derivatives of the displacement of the skin points and the points of the rib structure, respectively.

We reduce the number of scalar undetermined coefficients in the beam (2) by a factor of eight: three coordinates of the vector $u_{0}(z, \varphi)$, three coordinates of the vector $\psi^{-}$and two independent coordinates of the vector $\psi^{+}$.

The displacement of points on the surface of the reduction is determined as $u, v, w$, where $u$-displacement along the $s$ 'axis; $v$ and $w$ - displacements along the axes $t^{\prime}, n^{\prime}$, respectively. Linear displacements of the reduction surface along the axes $\left(s^{\prime}, t^{\prime}, n^{\prime}\right)$ determine $u_{0}(z, \varphi)$ in (2). They are functions of two coordinates:

$$
u_{s}{ }^{\prime}=u_{s^{\prime}}(z, \varphi), u_{t^{\prime}}=u_{t^{\prime}}(z, \varphi), u_{n^{\prime}}=u_{n^{\prime}}(z, \varphi) .
$$

From the joint deformation of the skin and the rib structure, we define:

- coordinates of a vector $\psi$ - as angles of rotation of the cross-sections of the edges relative to the axes $s^{\prime}, t^{\prime}, n$, in the form of $\theta_{s^{\prime}}, \theta_{t^{\prime}}, \theta_{n^{\prime}}$,

- coordinates of a vector $\psi^{+}$as the angles of rotation of the geometric normal of the skin from the initial state to the deformed in the form of $\theta_{s^{\prime}}^{\prime}=\partial w / \partial s^{\prime}$ and $\theta_{t^{\prime}}^{\prime}=$ $\partial w / \partial t$.

Thus, the eight functions of two coordinates $z$ and $\varphi$ uniquely determine the displacement field of the mesh structure with the shell:

- $\quad u_{s},(z, \varphi), u_{t},(z, \varphi), u_{n},(z, \varphi)$ - linear displacements of the reduction surface;

- $\theta_{s},(z, \varphi), \theta_{t^{\prime}}(z, \varphi), \theta_{n^{\prime}}(z, \varphi)$ - angles of rotation of the beam cross-section in the rib structure;

- $\theta_{s}^{\prime}(z, \varphi), \theta_{t^{\prime}}^{\prime}(z, \varphi)$ - angles of rotation of the geometric normal to the skin.

When examining a shell without skin, the angles of rotation of the geometric normal to the skin $\theta_{s}^{\prime},(z, \varphi), \theta_{t}^{\prime},(z, \varphi)$ introduced above will be absent.
Thus, the basic functions were constructed which ensure the compatibility of the movements of the ribs and the skin at the nodes on the reduction surface. The constructed model has the following properties:

- the movements of the ribs and the skin are coincident on the lines of the reduction surface that run parallel to the axes of the ribs (the lines of reduction for the ribs);

- deformation of the skin corresponds to the classical hypothesis of Kirchhoff-Love;

- $\quad$ each edge is represented as a set of short beams for which Tymoshenko's hypothesis is accepted;

- the movement of the spiral and annular ribs at their intersections is continuous;

- the stresses acting along the normal to the surface of the reduction are zero.

\section{DISCRETE MODELING}

Each constructive element is given by a corresponding set of finite elements for constructing a discrete mesh shell model. Spiral, annular ribs and frames are represented in the form of beams - Timoshenko beam (Fig. 4b). The movements are approximated by a one-dimensional Hermitian polynomial of the third order. The skin was represented in the form of triangular plates - Zenkevich-Argiris [14] plate (Fig. 4a). The displacements in the skin are approximated by an incomplete cubic polynomial.

Calculation of the stress-strain state of an anisogrid latticeshell structure is performed by the finite element method in variational formulation according to the Lagrange principle.

The local coordinate system $(s, t, n)$ is selected for each type of finite element. The origin of the coordinate system is at the point of the projection of the center of the element onto the surface of the reduction. Variable variables are the nodal values of the movements' functions on the reduction surface.

Thus, the discrete model of static deformation of the reticulate shell of a regular and irregular structure allows to ensure the compatibility of deformation of the skin and rib elements on their reduction lines. The model describes the static deformation of a net design without skin as a special case.

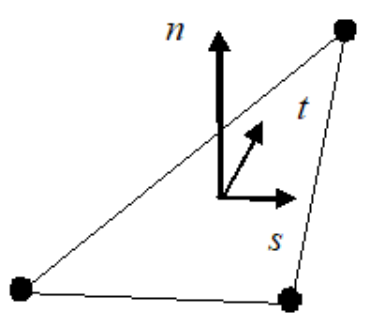

a)

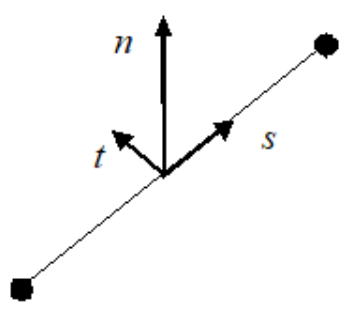

б)
Fig. 4. Finite elements: $\mathrm{a}$ - for the plate, $\mathrm{b}$ - for the beam

The most common form of loading of mesh structures is eccentric compression along the axis of the shell. The load can 
be represented as a uniform or uneven distribution of the running force at the upper edge, and also the application of the concentrated force and moments to the "hard" node.

\section{CONSTRUCTION OF A DISCRETE MODEL AND CALCULATION OF THE DEFORMED STATE OF THE AIRCRAFT COMPARTMENT}

We apply the models described above for calculating the stress-strain state of real spacecraft compartments. The design of the aircraft compartment is a finned shell in the form of a truncated cone. The shell has a double-layered skin and finning, which consists of 160 pairs of spiral ribs and 21 circular ribs. The circular edges are paired for technological reasons. The distance between the axes of pairs of circularly edges (the height of the rhombic cells) is $2 \cdot l_{h}$. The distance between the ribs inside the pair is $0.45 \cdot l_{h}$. The distance between the spiral ribs along the circumference is $1.483 \cdot l_{h}$ on average in height.

The height of the construction is $44.884 \cdot l_{h}$, the circumference along the leading edge is $237.02 \cdot l_{h}$, along the trailing edge $234.72 \cdot l_{h}$. The shell is supported by two rectangular cross-section frames. The shell has three cutouts and three hatches, which are covered with covers. The width of the section of the front reinforcing frame is $1.41 \cdot 1 l_{h}$, the width of the cross section of the back frame is $1.22 \cdot l_{h}$. The frames are reinforced with layers of skin and tape winding, which is made on top of the skin.

All cutouts are reinforced with edging of variable thickness. Edging is divided into three zones by their thickness. Hatches' covers in shape coincide with the hatches and have a thickness of $0.094 \cdot l_{h}$. Stringers of covers are made in the form of equilateral corners with a shelf width of $0.47 \cdot l_{h}$. The back edge of the shell is connected to the ring, which is fixed on 20 supports. The ring consists of a wall, front and rear shelves. The wall is made in the form of a cylindrical shell, whose height is $3.76 \cdot l_{h}$. The shelves are circular ribs.

Stresses were calculated by loading along the leading edge with a compressive force $P=1 \mathrm{MN}$ and bending moment $M=$ $12 \mathrm{P} \cdot \mathrm{N} \cdot \mathrm{m}$, for the purpose of studying the stress concentration.

The segments of the spiral and annular ribs and frames were split into an equal number of elements along the length, when a finite element model. The lengths of the finite elements of the annular edges turn out to be smaller than the spiral ones. The paneling was divided into triangular finite elements with the same set of nodes. The topological scheme of the grid of triangular elements is shown in Fig. 5. Edging is divided into zones in the finite element model: the inner part is a three-layer package of layers of inner, middle and outer fringing, the middle part is a two-layer package of middle and outer fringing, and the outer part - only a layer of outer rim.
Calculations with the breakdown of the segments of edges into a different number of finite elements were carried out in order to confirm the grid convergence of the numerical solution.

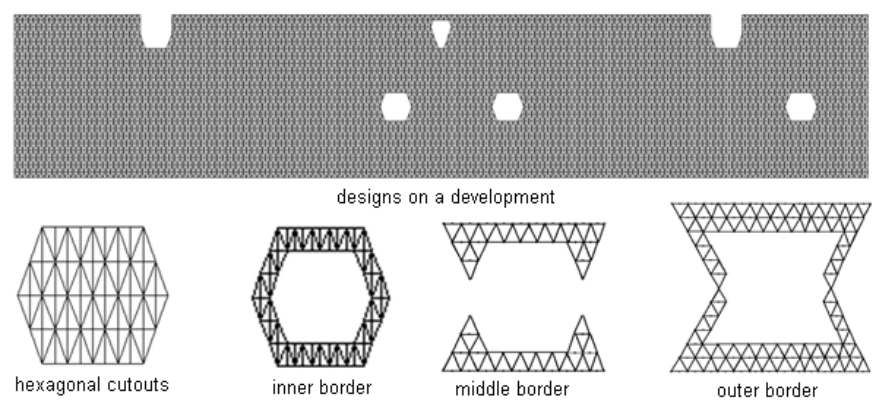

Fig. 5. Finite element model of construction

Calculation of the stress-strain state of the compartment was carried out in the program complex "Composite NK Anisogride" [15]. The overall dimensions of the structure under consideration are normalized by its height and the circumference of the trailing edge to represent the results. The normalized height of the compartment y' varies from 0 to 1 , and the normalized edge length $x^{\prime}$ from $-\pi$ to $\pi$.

\section{A. Stresses in the shell}

The stress fields $\sigma_{s}$ along the generatrix in the outer layer of the skin are shown in Fig. 6. The greatest stresses os are reached at the edges of the cut-outs, on top and bottom of the hatchways of the manholes. The maximum value of the compressive stress $\sigma$ s along the generator in this layer is equal to $-177.89 \mathrm{MPa}$.

The circumferential stresses $\sigma_{t}$ in the outer layer are distributed throughout the region more evenly, without pronounced differences. The stress values are in the range from -34.01 to $21.28 \mathrm{MPa}$ in most of the region. The zones of the largest stretches are located at the edges of the first and third hatches, at a height $y^{\prime}=0.5$. The maximum value of circumferential stresses is $353.92 \mathrm{MPa}$ in these regions.

The values of tangential stresses $\tau_{s t}$ grow around cutouts. The areas of maximum positive stress values $\tau_{s t}$ are located on the right at the edge of the first and second notches. Zones of the greatest compression are concentrated on the edge of the hatches. They alternate with stretching zones. The maximum values of the compressive stresses $\tau_{s t}$ in the outer skin layer are $-25.89 \mathrm{MPa}$. The values of the greatest tangential stresses $\tau_{s t}$ are $26.18 \mathrm{MPa}$. 
a)
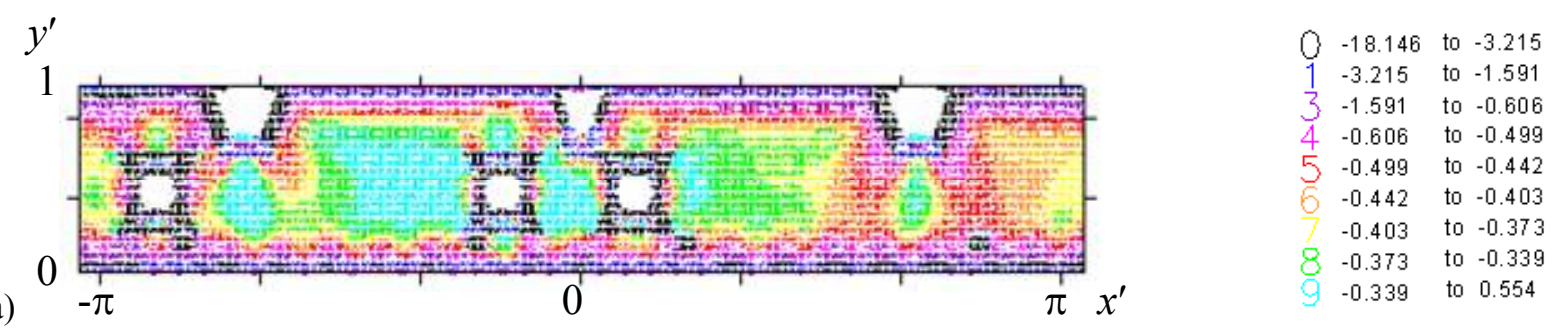

$y^{\prime}$

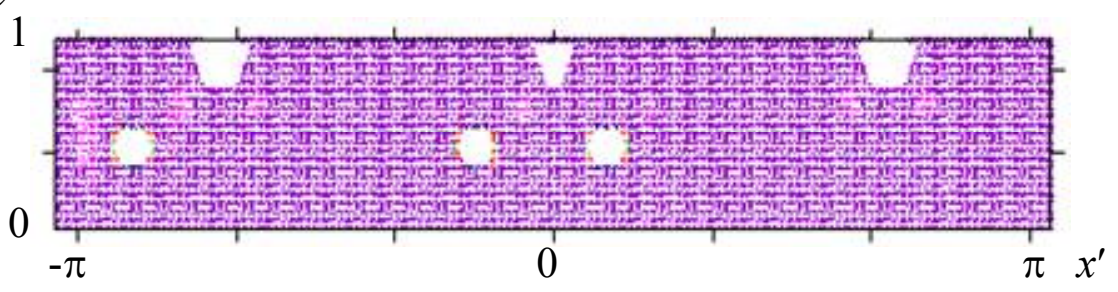

b)

$\begin{array}{lll}0 & -14.781 & \text { to }-9.129 \\ 1 & -9.129 & \text { to }-3.478 \\ \frac{1}{3} & -3.478 & \text { to } 2.173 \\ 4 & 2.173 & \text { to } 7.824 \\ 5 & 7.824 & \text { to } 13.476 \\ 6 & 13.476 & \text { to } 19.127 \\ & 19.127 & \text { to } 24.779 \\ 8 & 24.779 & \text { to } 30.430 \\ 9 & 30.430 & \text { to } 36.09\end{array}$

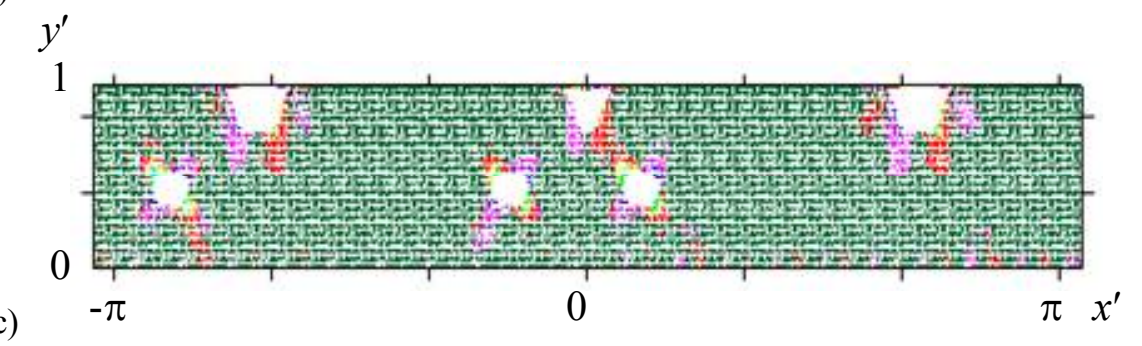

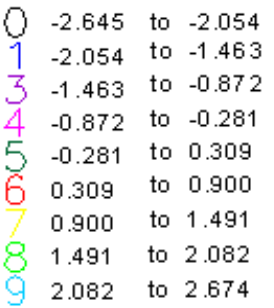

Fig. 6. Stress distributions $\left(10^{-1} \cdot \mathrm{MPa}\right)$ in the outer skin layer: $\mathrm{a}-\sigma_{s} ; \mathrm{b}-\sigma_{t} ; \mathrm{c}-\sigma_{s t}$

\section{B. Stresses in the ribs}

Distributions of longitudinal stresses $\sigma_{s}$ in spiral and annular ribs in Fig. 7.

The zones of maximum elongation of the spiral ribs are located in the region of the greatest loads between the second and the first notches. The areas of maximum stretching fall on the boundaries of the first and second notches: in the case of spiral ribs with a right slope - on the right side.

The area of greatest compression extends from the left border of the second cutout to the adjacent zone of the right border of the first cutout. A vast area around the first hatch enters the area of greatest compression. The stress values in it are on the order of 117.69-93.16 MPa.

The zones of least stresses are located on the top and bottom of the first and second hatches. They include stretch

a)

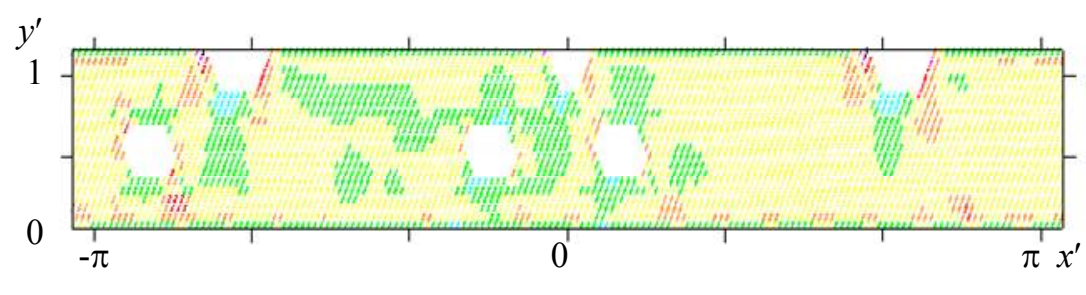

points. The same areas are adjacent to the bottom of all the cutouts. The stress values in these zones range from -16.67 to $16.67 \mathrm{MPa}$.

The ring fins of the structure are generally susceptible to stretching, but there are also areas of compression. The zones of greatest compression are located around the cut-outs and manholes. The area of increased stretching is located between the second and the first notches in the zone of the greatest loads in the annular ribs (the middle part of their cross section). The values of the component $\sigma_{s}$ vary from 21.57 to $31.38 \mathrm{MPa}$. The stresses $\sigma_{s}$ take the maximum positive values along the edges of the first and second notches in the circular ribs (71.59 MPa). 
b)

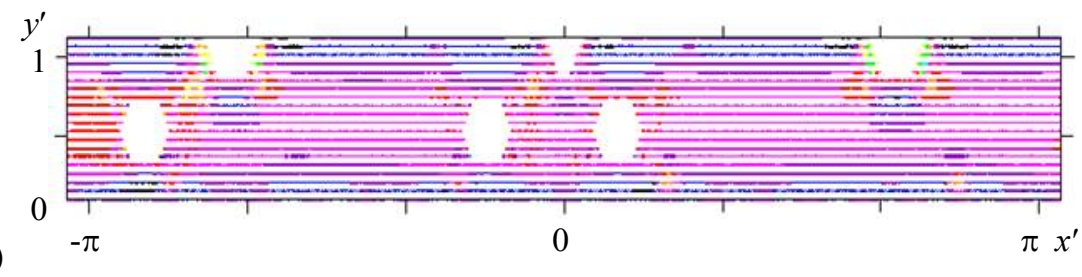

$\begin{array}{lll}0 & -1.855 & \text { to }-0.838 \\ 1 & -0.838 & \text { to } 0.179 \\ 3 & 0.179 & \text { to } 1.196 \\ 4 & 1.196 & \text { to } 2.214 \\ 5 & 2.214 & \text { to } 3.231 \\ 8 & 3.231 & \text { to } 4.249 \\ 4.249 & \text { to } 5.266 \\ 8 & 5.266 & \text { to } 6.284 \\ 9 & 6.284 & \text { to } 7.302\end{array}$

Fig. 7. Stress distributions $\sigma_{s}\left(10^{-1} \cdot \mathrm{MPa}\right)$ : a - in spiral ribs with a slope to the right, $\mathrm{b}$ - in annular ribs

Analysis of the calculated stress fields allows one to get an idea of the zones that are subject to the greatest loading. We can also draw a conclusion about the behavior of the structure when the compressive force is applied to it as a whole on the basis of this analysis.

\section{FORMAL FORMULATION OF OPTIMAL AND RATIONAL DESIGN PROBLEMS, ALGORITHM FOR SOLVING THE PROBLEM}

Composite material is formed simultaneously with the manufacture of the product itself with the modern technology of manufacturing anisogrid lattice shells. Since the properties of the reinforced material depend significantly on technological factors, it is difficult to accurately assign the physicomechanical properties to the composite material during manufacture. Therefore, a significant spread in the values of the physical and mechanical characteristics of the material can be observed in a series of similar products. Technological design imperfections have a significant effect on the physical and mechanical characteristics.

The foregoing allows us to consider the mathematical model of static deformation of the power structure at the level of the "black box" [16]. In this model, the internal structure of the modeled object is not disclosed and the reasons for the reaction of the object to the applied impacts are fixed.

$$
q=K(p) r,
$$

where $q-$ is the vector of state variables (displacements, stresses and deformations);

$r-$ is the vector of the variable effects (applied forces);

$K-$ is the model operator that maps the elements of the action space to the state space elements;

$p-$ is a vector of model parameters that includes the "internal" characteristics of the modeled object (physical and mechanical constants of materials, geometric dimensions of the structure, dimensions of sections of structural elements, etc.).

The problem of rational design design is to determine the values of the structural parameters of the model that satisfy the initially specified constraints, without the condition of a minimum of the optimization criterion. Dimensions of the structure (its radius and height), a given number of spiral and / or annular ribs, etc., as well as limitations in strength, stiffness and stability are considered as limitations of structural parameters for lattice shells.

The problem of rational design design can be written as follows.

\section{It is known:}

- initial values of the structural parameters of the model $X_{0}$,

- a vector of variable effects $r$,

- model of the reaction of the structure to the effects $q=K(p) r$.

It is required to determine: the structural parameters of the model $X \subseteq p$, under which the structural parameters of the construction $F(p) \geq 0$ and the state parameters $\Phi(q) \geq 0$ are satisfied.

The solution of this problem in the form of a vector of structural parameters of the model is not unique. This allows us to refine the boundaries of acceptable values of structural parameters and use them in the task of optimizing the design by mass.

The task of optimizing the structure by mass is formulated as follows: find the values of the structural parameters $p$ that provide a minimum of the quality criterion (mass), provided that the state parameters q satisfy the system of specified constraints (strength, rigidity and stability, etc.).

Formally, the problem of optimizing the construction by mass based on the model (4) can be put in the following form.

\section{It is known:}

- initial values of the structural parameters of the model $X_{0}$,

- a vector of variable effects $r$,

- model of the reaction of the structure to the effects $q=K(p) r$.

It is required to determine: the structural parameters of the model $\mathrm{X} \subseteq \mathrm{p}$, for which the constraints of the structural parameters $F(p) \geq 0$ and the constraints of the state parameters $\Phi(q) \geq 0$ are satisfied, which ensure the minimum of the objective function $M(p) \rightarrow$ min. The mass of the structure is chosen as the objective function $M(p)$.

Strength, stiffness and stability of structures act as state variables, when the problems of optimizing lattice anisogrid structures and rational design are solved [17, 18]. The mathematical model of joint deformation of structural elements and a discrete approach in the development of finite element models can be used to calculate the variable states of anisogrid structures. 


\section{CONCLUSION}

The use of a discrete approach and mathematical modeling (the model of joint deformation of all structural elements) allows solving the problems associated with ensuring the strength of mesh anisogrid structures: rational design, estimation of actual strength parameters and rigidity when finalizing experimental designs, and optimal design problems. This approach makes it possible to investigate the behavior of the mesh anisogrid constructions of an irregular structure.

\section{REFERENCES}

[1] Yu.S. Solomonov, V.V. Vasilyev and V.P. Georgievsky, "Composite materials in rocket and aerospace engineering" // Proceedings of Moscow. Institute of Heat Engineering, 2006, Vol. 8, part 1, pp. 7-25.

[2] V.V. Vasilyev, V.A. Barynin, A.F. Razin, S.A. Petrokovsky and V.I. Khalimanovich, "Anisogrid composite structures - development and application to space technology'. Composites and nanostructures [Композиты и наноструктуры]. 2009, № 3, pp. 38-50.

[3] V.A. Barynin, V.A. Bunakov, V.V. Vasilyev, B.G. Mayorov and A.F. Razin, "Composite mesh constructions: a review". Vopr. obor. engineering [Вопр. обор. техники], ser. 15, 2001, vol. 1 (123) - 2 (124), pp. 9-16.

[4] V. V. Vasiliev, Mechanics of constructions from composite materials [Механика конструкций из композиционных материалов]. М .: Mechanical Engineering, 1988, 272 p.

[5] V.V. Vasiliev, "Shells and plates of composite material with a spatial reinforcement scheme" [Оболочки и пластинки из композиционного материала с пространственной схемой армирования]. Design, calculation and testing of structures from composite materials.1982, Issue 9, pp.83-90.

[6] V.V. Vasilyev, A.A. Skleznev, "Longitudinal and flexural vibrations of the mesh composite transitional compartment of the spacecraft." Mechanics of composite materials and structures 2009, vol.15, N 2, pp. 242-255.

[7] V.V. Vasiliev, V.A, Bunakov, "Design of mesh composite cylindrical shells, compressed in the axial direction" [Проектирование сетчатых композитных цилиндрических оболочек, сжатых в осевом направлении]. Mechanics of constructions from composite materials [Механика конструкций из композиционных материалов]. 2000, № 2 , pp. $68-77$.

[8] V.V. Vasiliev and oth., Mesh composite compartment for connecting a launch vehicle with a spacecraft .Flight. 1999, №9, pp. 44-47.

[9] V.V. Vasiliev, A.V. Lopatin, The theory of reticulated and reinforced composite shells. Mechanics of constructions from composite materials. 1984, pp. 31-36.

[10] K.-Yu. Bathe Methodykonechnykhelementov [Finite Elements Methods].Transl. into Russian. Moscow, FIZMATLIT Publ., 2010, $1024 \mathrm{p}$.

[11] T.V. Burnysheva, V.O. Kaledin, "Comparison of discrete and continual approach to calculation of a stressed state of network capsulate designs at a static loading” Nauchno-tekhnicheskiy vestnik Povolzh'ya, 2011, № 4, pp. 113-115.

[12] S.A. Ambartsumian, Theory of Anisotropic Plates: Strength, Stability and Oscillations.M.: Science, 1987, 360 p.

[13] S.P. Timoshenko, Mechanics of Materials. SPb.: Publishing House "Lan", 2002, 672 p.

[14] O. Zenkevich, Finite Element Method in Engineering. Moscow: Mir, $1975,544 \mathrm{p}$

[15] V.O. Kaledin, Ya.S. Kryukova, N.V. Nagaytseva and E.V. Ravkovskaya "Software system for algorithmization numerical solution of problems of continuum mechanics" Izvestiya Altayskogo gosudarstvennogo universiteta. 2014, №. 1-1(81), pp.161-164.

[16] O.K. Kaznacheeva, V.O. Kaledin, Identification of parameters of elasticity and rigidity of structures from reinforced materials. Novocherkassk, LIC, 2012, 135 p.
[17] T.V. Burnysheva, O.A. Steinbreher, "Rational projection of the thickness of the edging of the hatches of the design of the spacecraft's compartment”. Engineering Journal: Science and Innovation, 2017, № 9. http://dx.doi.org/10.18698/2308-6033-2017-9-1671

[18] O.A. Steinbreher, T.V. Burnysheva, "Solution of the problem of parametric optimization of a mesh cylindrical structure".Engineering Journal: Science and Innovation, 2017, № 10 . http://dx.doi.org/10.18698/2308-6033-2017-10-1688 